\title{
Iterative scheme for computing exactly the total field propagating in dielectric structures of arbitrary shape
}

\author{
Olivier J. F. Martin \\ IBM Research Division, Zurich Research Laboratory, 8803 Rueschlikon, Switzerland
}

Alain Dereux

Institute for Studies in Interface Sciences, Faculté Universitaire Notre-Dame de la Paix, 61, Rue de Bruxelles, 5000 Namur, Belgium

\section{Christian Girard}

Laboratoire de Physique Moléculaire Unité Associée Centre de la Research Scientifique 772, Université de Franche Comté, 25030 Besançon Cedex, France

\begin{abstract}
Received March 25, 1993; revised manuscript received September 27, 1993, accepted September 28, 1993
We present a new approach to the computation of an electrical field propagating in a dielectric structure. We use the Green's-function technique to compute an exact solution of the wave equation. No paraxial approximation is made, and our method can handle any kind of dielectric medium (air, semiconductor, metal, etc.). An original iterative numerical scheme based on the parallel use of Lippman-Schwinger and Dyson's equations is demonstrated. The influence of the numerical parameters on the accuracy of the results is studied in detail, and the high precision and stability of the method are assessed. Examples for one and two dimensions establish the versatility of the method and its ability to handle structures of arbitrary shape. The application of the method to the computation of eigenmode spectra for dielectric structures is illustrated.
\end{abstract}

\section{INTRODUCTION}

Since the original work of Feit and Fleck ${ }^{1-3}$ significant progress has been made in the domain of the beampropagation method (BPM). And since the assessment of its applicability 4,5 this method has certainly become one of the most widely used modeling tools for integrated optics.

Improvements in the original Fourier-transform-based algorithm with the use of either finite differences ${ }^{6,7}$ or finite elements, ${ }^{8,9}$ have increased its efficiency. Alternative approaches, such as those based on the method of line ${ }^{10}$ or the finite-difference time-domain method, ${ }^{11}$ have also been proposed.

In the past few years the development of the BPM has taken two directions. First, extensive efforts have been devoted to overcoming the limitation of the paraxial approximation ${ }^{12-14}$ and to deriving an exact solution of the scalar-wave equation. ${ }^{15}$ Second, semivectorial methods have been proposed ${ }^{16-18}$ that can treat the various components of the electrical field simultaneously but not the coupling among these components. More recently, fully vectorial methods have been demonstrated ${ }^{19-24}$ that can model such vectorial effects as polarization within the limited framework of the paraxial approximation.

Because the paraxial approximation is not well founded for numerous devices of integrated optics and because these devices make use of vectorial effects such as polarization splitting, there is a quest for a nonparaxial method that can treat vectorial fields propagating in arbitrary dielectric media. We propose an original approach to this problem and demonstrate a new method for solving exactly the wave equation for arbitrary dielectric media. For the sake of simplicity we present the method for scalar fields in one- and two-dimensional media, but it can also be generalized to vectorial fields in three-dimensional media, as is discussed at the end of Section 2.

Our method is based on the Green's function technique. This formalism has been shown to be very powerful for studying the eigenmodes of dielectric structures invariant along the propagation direction ${ }^{25-27}$ as well for describing nonradiative energy transfers occurring in near-field microscopy. ${ }^{28,29}$ To the best of our knowledge, however, it has not yet been used to compute the field propagating in an arbitrary structure.

In Section 2 we develop the basic formalism and explain the algorithm that we used. In Sections 3 and 4 we give one- and two-dimensional examples that assess the accuracy and applicability of the method. Finally, in Section 5 we summarize our results.

\section{NUMERICAL METHOD}

The objective of our method is to find a solution $\psi(\mathbf{r})$ of the scalar-wave equation

$$
\nabla^{2} \psi(\mathbf{r})+\omega^{2} \mu_{0} \varepsilon_{0} \varepsilon(\mathbf{r}) \psi(\mathbf{r})=0
$$

for an arbitrary complex dielectric medium $\varepsilon(\mathbf{r})$ of homogeneous permittivity $\mu_{0}$. In Eq. (1) we assume the usual time dependence $\sim \exp (-i \omega t)$ for the electrical field $\psi(\mathbf{r})$. Such a scalar field describes, for example, the transverse electric modes propagating in a slab waveguide. ${ }^{30}$ 


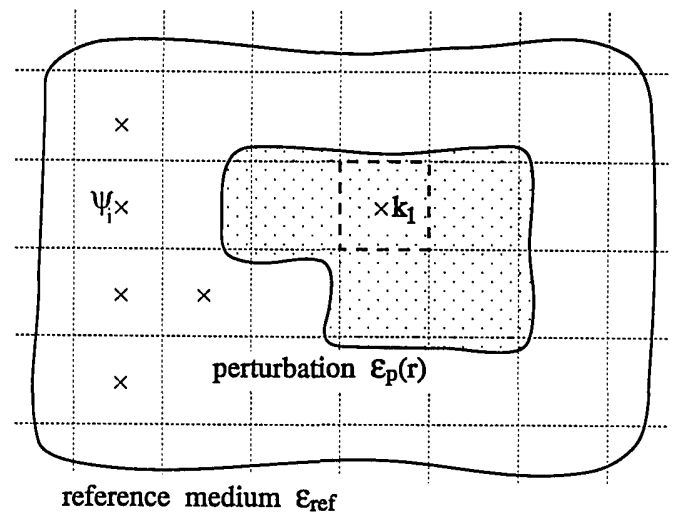

Fig. 1. Geometry used in the model. The dielectric medium for which we seek a solution of the wave equation can be split into a homogeneous reference medium $\varepsilon_{\text {ref }}$ and an arbitrary perturbation $\varepsilon_{p}$ embedded in the reference medium. The discretization grid is also shown.

The only assumption that we make regarding the dielectric medium is that it can be split into two parts (Fig. 1): a homogeneous reference medium of dielectric constant $\varepsilon_{\text {ref }}$ and a perturbation $\varepsilon_{p}(\mathbf{r})$ confined to the inside of the reference medium. Hence the dielectric function of the system can be written as

$$
\varepsilon(\mathbf{r})=\varepsilon_{\mathrm{ref}}+\Delta \varepsilon_{p}(\mathbf{r}),
$$

with $\Delta \varepsilon_{p}(\mathbf{r})=\varepsilon_{p}(\mathbf{r})-\varepsilon_{\text {ref }}$. This assumption can easily be fulfilled where both the reference medium and the perturbation depend on the problem investigated. For example, if we study an optical fiber in vacuum, the reference medium is a vacuum and the perturbation describes the fiber. For a ridge waveguide the reference medium is the substrate and the perturbation is the ridge. Note that $\varepsilon_{\text {ref }}$ is simply the value of the homogeneous reference medium; it is not, as in the BPM, the value associated with the wave number of the propagated field.

It is important to point out that no restrictions are imposed on the perturbation $\varepsilon_{p}$; it can have any shape and describe any kind of dielectric medium. Absorption can easily be introduced into the imaginary part of $\varepsilon_{p}$, while a metallic perturbation can be accounted for with the use of negative value for the real part of $\varepsilon_{p}$ (see Fig. 6 below). Furthermore, no assumptions are required regarding the difference between $\varepsilon_{\text {ref }}$ and $\varepsilon_{p}$, as is the case for the BPM.

If we know a solution $\psi^{0}(\mathbf{r})$ of Eq. (1) corresponding to the homogeneous medium $\varepsilon_{\text {ref }}$ without perturbation, we can compute a solution for the perturbated system using the Lippman-Schwinger equation, ${ }^{31}$

$$
\psi(\mathbf{r})=\psi^{0}(\mathbf{r})+\int_{\text {perturbation }} \mathrm{d} \mathbf{r}^{\prime} G^{0}\left(\mathbf{r}, \mathbf{r}^{\prime}\right) V\left(\mathbf{r}^{\prime}\right) \psi\left(\mathbf{r}^{\prime}\right),
$$

where we have introduced the dyadic Green's operator $G^{0}\left(\mathbf{r}, \mathbf{r}^{\prime}\right)$ associated with the homogeneous reference system. The function $V(\mathbf{r})$ describes the perturbation and is defined by

$$
V(\mathbf{r})=-k_{0}^{2} \Delta \varepsilon_{p}(\mathbf{r}),
$$

where $k_{0}^{2}$ is the wave number in vacuum:

$$
k_{0}^{2}=\omega^{2} \varepsilon_{0} \mu_{0} .
$$

The integration domain in Eq. (3) is limited to the perturbation. Thus this equation is implicit for all points located inside the perturbation. Once the field inside the perturbation has been computed, it can be generated explicitly for any point of the reference medium with Eq. (3).

We now define a grid over our system (see Fig. 1). The discretization procedure is identical in one, two, or three dimensions; for clarity we use only one figure to mark the position of a mesh, and we designate its volume by $\Delta j$. A regular grid is not required, and for many applications a variable Gaussian grid may be advantageous. ${ }^{32}$ We suppose that the discretized system contains $N$ meshes from which $N_{p}$ describe the perturbation $\left(N_{p} \leq N\right)$. We designate the discretized field, the Green's operator, and the perturbation by $\psi_{i}, G_{i, j}$, and $V_{i}$, respectively.

The discretized form of Eq. (3) leads to a large system of linear equations for the electrical field:

$$
\psi_{i}=\psi_{i}^{0}+\sum_{k=1}^{N_{p}} G_{i, k}^{0} V_{k} \Delta_{k} \psi_{k}
$$

The direct numerical resolution of Eq. (6) is particularly time consuming and may lead to numerical instabilities because of the singular behavior of $G_{i, i}^{0}$ (the renormalization of the self-field is discussed at the end of this section). To avoid these problems we propose an iterative scheme for computing the electrical field. For this purpose we introduce Dyson's equation, ${ }^{31}$

$$
\begin{aligned}
G\left(\mathbf{r}, \mathbf{r}^{\prime}\right)= & G^{0}\left(\mathbf{r}, \mathbf{r}^{\prime}\right) \\
& +\int_{\text {perturbation }} \mathrm{d} \mathbf{r}^{\prime \prime} G^{0}\left(\mathbf{r}, \mathbf{r}^{\prime \prime}\right) V\left(\mathbf{r}^{\prime \prime}\right) G\left(\mathbf{r}^{\prime \prime}, \mathbf{r}^{\prime}\right)
\end{aligned}
$$

and its discretized form,

$$
G_{i, j}=G_{i, j}^{0}+\sum_{k=1}^{N_{p}} G_{i, k}^{0} V_{k} \Delta_{k} G_{k, j}
$$

Dyson's equation is the counterpart of the Lippman-Schwinger equation for operators. It yields Green's function $G\left(\mathbf{r}, \mathbf{r}^{\prime}\right)$ of the perturbated system, given Green's function $G^{0}\left(\mathbf{r}, \mathbf{r}^{\prime}\right)$ of the homogeneous reference system. In our iterative procedure we use both equations in parallel, as described in the next paragraph.

Instead of computing the solution of Eq. (1) corresponding to the whole perturbation, we first consider only one mesh of the perturbation, say, $k_{1}$ (see Fig. 1), and compute the field $\psi_{i}{ }^{1}$ and Green's function $G_{i, j}{ }^{1}$ corresponding to a homogeneous medium $\varepsilon_{\text {ref }}$ plus the infinitesimal perturbation $k_{1}$. Equations (6) and (8) become, respectively,

$$
\begin{aligned}
\psi_{i}{ }^{1} & =\psi_{i}{ }^{0}+G_{i, k_{1}}^{0} V_{k_{1}} \Delta_{k_{1}} \psi_{k_{1}}, \\
G_{i, j}^{1} & =G_{i, j}^{0}+G_{i, k_{1}}^{0} V_{k_{1}} \Delta_{k_{1}} G_{k_{1}, j}^{1} .
\end{aligned}
$$

Equations (9) and (10) require no resolution of a system of equations. They are first worked out for $i=k_{1}$, and this result is used to compute $\psi_{i}{ }^{1}$ and $G_{i, j}^{1}$ for $i \neq k_{1}$. This procedure is straightforward and very efficient from a numerical point of view.

We then consider a second mesh of the perturbation, say, $k_{2}$, and compute the corresponding field $\psi_{i}{ }^{2}$ and 


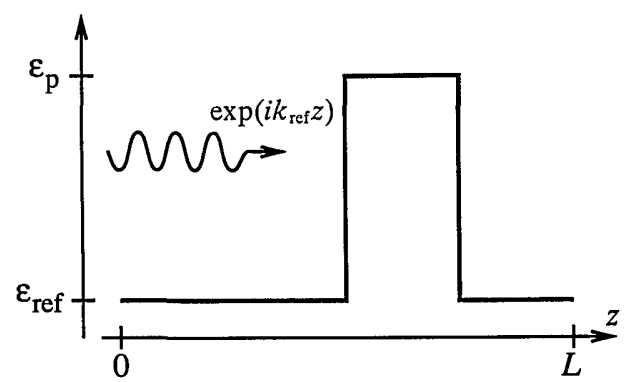

Fig. 2. Plane wave impinging upon a dielectric barrier. The reference medium $\varepsilon_{\text {ref }}$ corresponds to the fundamental level, whereas the perturbation $\varepsilon_{p}$ describes the barrier.

Green's function $G_{i, j}^{2}$, using the results of the preceding step:

$$
\begin{aligned}
\psi_{i}{ }^{2} & =\psi_{i}{ }^{1}+G_{i, k_{2}}^{1} V_{k_{2}} \Delta_{k_{2}} \psi_{k_{2}}, \\
G_{i, j}^{2} & =G_{i, j}^{1}+G_{i, k_{2}}^{1} V_{k_{2}} \Delta_{k_{2}} G_{k_{2}, j}^{2} .
\end{aligned}
$$

Applying this scheme successively to each mesh in the perturbation, we construct the field $\psi_{i}{ }^{N_{p}}$ corresponding to the whole perturbated system. It is interesting to note that at each step $l$ it is necessary to compute Green's function $G_{i, j}^{l}$ only for $j$ equal to the remaining perturbations. Therefore the number of operations and the size of the matrix $G_{i, j}$ required for each step decrease as the calculation proceeds. As we have mentioned, it is initially possible to limit the above-mentioned procedure to compute only the field inside the perturbation and to use this result to generate, at a later stage, the field outside the perturbation with Lippman-Schwinger equation (6).

The form of Green's functions corresponding to one- and two-dimensional media is given in Ref. 31. For a twodimensional medium, Green's function diverges for $\mathbf{r}=\mathbf{r}^{\prime}$. To overcome this problem we introduce a renormalization of Green's function. This procedure has been explained in detail by Yaghjian. ${ }^{33}$ It consists of integrating analytically $G^{0}(\mathbf{r}, \mathbf{r})$ over one mesh of the system and then using this value for $G^{0}(\mathbf{r}, \mathbf{r})$.

For a vectorial field $\psi(\mathbf{r})$ the algorithm is similar. In that case the discretized Green's function $\mathbf{G}_{i, j}$ is a $3 \times 3$ matrix, and each discretized Dyson's equation [Eqs. (10) and (12)] leads to a system of nine algebraic equations (one per $\mathbf{G}_{i, j}$ component) that must be solved at each computational step to produce $G_{k l, j}^{l}$. As for the LippmanSchwinger equation [Eqs. (9) and (11)], it becomes a system of three algebraic equations (one per field component).

Unfortunately, a von Neumann analysis ${ }^{34}$ cannot be applied to our method to assess its accuracy. We nevertheless show in Sections 3 and 4, using one- and two-dimensional examples, respectively, how the grid refinement influences the accuracy of the results.

\section{ONE-DIMENSIONAL EXAMPLES}

In this section we consider a square dielectric barrier (Fig. 2). For such a system the reference medium $\varepsilon_{\text {ref }}$ is the fundamental level, whereas the perturbation $\varepsilon_{p}(z)$ describes the barrier. When a plane wave

$$
\psi^{0}(z)=\exp \left(i k_{\mathrm{ref}} z\right)
$$

with a wave number corresponding to the reference medium impinges upon the barrier, one part is transmitted and one part is reflected, thus interfering with the incoming wave. This behavior is easily obtained with our method, as can be seen from Fig. 3, where we present the amplitude of the computed field. Three different cases are investigated: a nonabsorbing barrier $\left(\varepsilon_{p}=4.0\right)$, a barrier with limited absorption $\left(\varepsilon_{p}=4+i 1.0\right)$, and a barrier with strong absorption $\left(\varepsilon_{p}=4+i 4.0\right)$. For each case the reference medium is a vacuum and the wavelength of the incident wave is $\lambda_{0}=0.8 \mu \mathrm{m}$. The attenuation of the wave as a function of the absorption in the barrier is clearly visible in Fig. 3. Complementary results, such as the variation of transmission and reflection coefficients as a function of the barrier thickness, ${ }^{35}$ are also accurately reproduced with our method.

The advantage of this simple system is that an analytical solution $\phi(z)$ can easily be obtained. ${ }^{36}$ Thus the norm of the difference between numerical and analytical solutions allows the accuracy of the computational method to be evaluated:

$$
\|\psi-\phi\|^{2}=\int_{0}^{L} \mathrm{~d} z[\phi(z)-\psi(z)]^{*}[\phi(z)-\psi(z)] .
$$

When $\|\psi-\phi\|^{2}=0$ the agreement between analytical and numerical results is exact.

For a given wavelength and height of the barrier $\varepsilon_{p}$, $\|\psi-\phi\|^{2}$ depends on the mesh size $\Delta_{z}$ (Fig. 4). From least-squares fits to these data we find that $\|\psi-\phi\|^{2}$ is proportional to $\Delta_{z}$ at a power of 3 . The value of $\Delta_{z}$ necessary for achieving excellent agreement between numerical and analytical results also varies with $\varepsilon_{p}$ : a higher barrier requires a finer grid. But whatever the barrier is, it is possible to obtain extremely accurate results (Fig. 4).

The determinant parameter here is not simply the barrier height but the effective wavelength in the barrier $\lambda_{\text {eff }}=\lambda_{0}\left(\varepsilon_{p}\right)^{-1 / 2}$. This can be seen in Fig. 5, where we show $\|\psi-\phi\|^{2}$ as a function of the mesh size for different effective wavelengths. From these data it is clear that the mesh size that one requires to obtain a given accuracy is inversely proportional to $\lambda_{\text {eff }}$. For any value of the

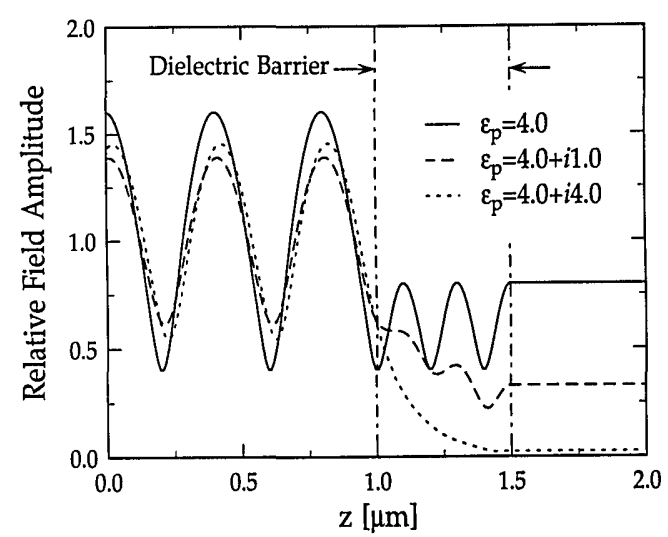

Fig. 3. Amplitude of the computed field corresponding to the geometry depicted in Fig. 2, normalized to the incident field. Three dielectric barriers $\varepsilon_{p}$ with different absorption values are investigated. The reference medium is a vacuum, and the wavelength of the incoming wave is $\lambda_{0}=0.8 \mu \mathrm{m}$. The mesh size for the calculation was $\Delta_{z}=0.01 \mu \mathrm{m}$. 


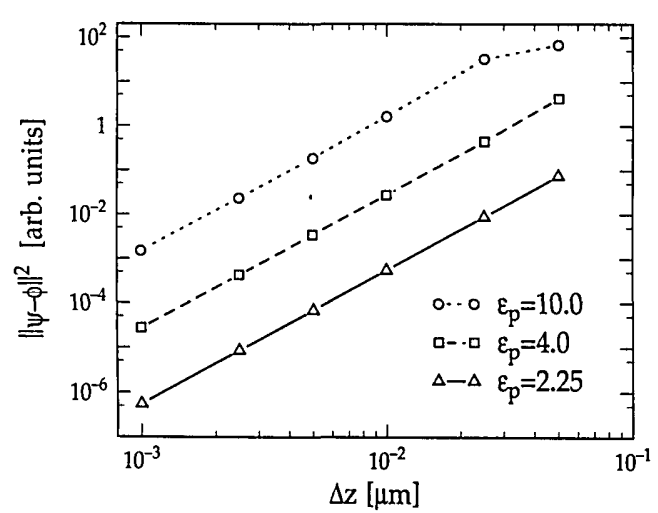

Fig. 4. Norm of the difference between numerical and analytical results as a function of the mesh size $\Delta_{z}$. The corresponding geometry is depicted in Fig. 2. Three barriers of $1-\mu \mathrm{m}$ thickness and varying dielectric constant $\varepsilon_{p}$ are investigated. The wavelength in a vacuum is $0.8 \mu \mathrm{m}$.

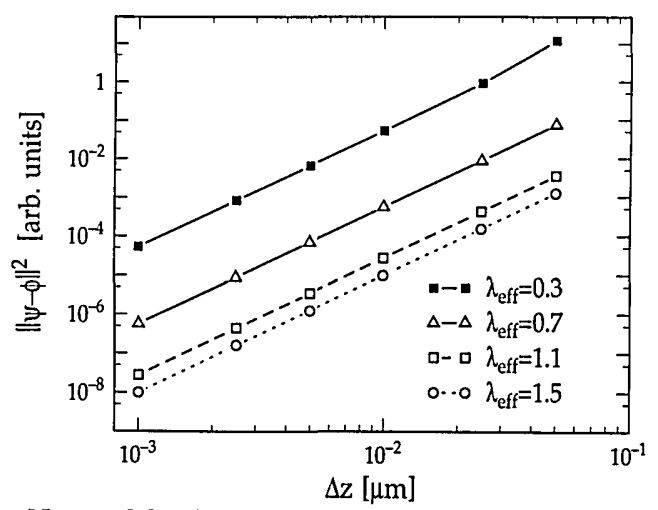

Fig. 5. Norm of the difference between numerical and analytical results as a function of the mesh size $\Delta_{z}$ for different effective wavelengths in the barrier depicted in Fig. 2.

effective wavelength, $\|\psi-\phi\|^{2}$ is proportional to $\Delta_{z}{ }^{3}$ (Fig. 5).

Our method is also perfectly stable; an excessively small grid does not lead to numerical instabilities but always enhances precision. We believe that this stability is due to the fact that at each step we consider only one perturbation and therefore must handle only one renormalized element $G^{0}(\mathbf{r}, \mathbf{r})$ at a time. The discretization procedure being exactly identical in one, two, or three dimensions, we can assume a similar behavior of the accuracy of the algorithm for two- and three-dimensional systems.

\section{TWO-DIMENSIONAL EXAMPLES}

We consider first the interaction of a plane wave in vacuum $\left(\varepsilon_{\text {eff }}=1\right)$ with a microscopic square dielectric pad of homogeneous dielectric constant $\varepsilon_{p}=2$. The amplitude of the corresponding computed field is depicted in Fig. 6(a). The wavelength of the incoming wave is $1 \mu \mathrm{m}$. As for a one-dimensional barrier, some of the initial field is reflected and interferes with the incoming wave. Owing to the limited lateral extension of the obstacle, a diffraction pattern appears in the right-hand part of Fig. 6(a). The fine structure of the electrical field in the pad and in its vicinity is also resolved; this information is useful in connection with experiments such as those in scanning near-field optical microscopy. ${ }^{37}$
If we use a gold aggregate $\left(\varepsilon_{p}=-42.8+i 1.3\right)$, the field pattern is completely different [Fig. 6(b)]. Most of the incoming wave is reflected, whereby the metallic pad acts as a point source emitting in the negative $z$ direction (note the curvature of the phase fronts on the interference pattern). The field vanishes in the metal, and the skin effect ${ }^{38}$ is accurately reproduced at its surface. This emphasizes the versatility of our method.

In Fig. 7 we describe the interaction of the same plane wave with a metallic plate $\left(\varepsilon_{p}=-42.8+i 1.3\right)$ of $0.1-\mu \mathrm{m}$ thickness. The plate forms a $45^{\circ}$ angle with the propagation direction of the incoming wave. Most of the in-
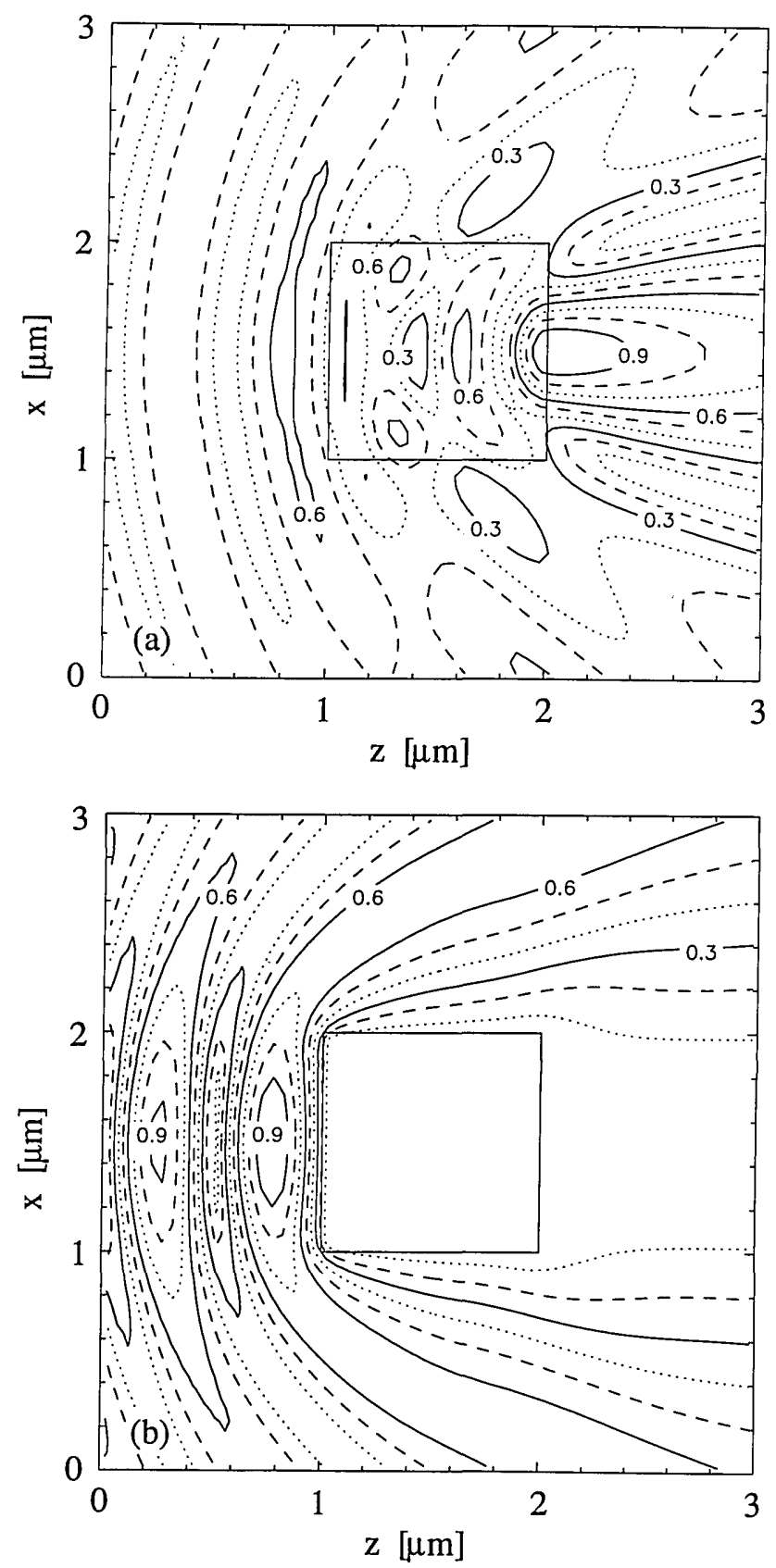

Fig. 6. Interaction of a plane wave in a vacuum $\left(\varepsilon_{\mathrm{ref}}=1\right)$ (a) with a diclectric pad $\left(\varepsilon_{p}=2\right)$ and (b) with a gold pad $\left(\varepsilon_{p}=-42.8+i 1.3\right)$. The spacing between the isoamplitude curves is $10 \%$ of the corresponding maximum-minimum amplitude range. The wavelength of the incoming wave is $\lambda_{0}=1 \mu \mathrm{m}$, and the mesh size is $\Delta_{x}=\Delta_{z}=0.05 \mu \mathrm{m}$. 


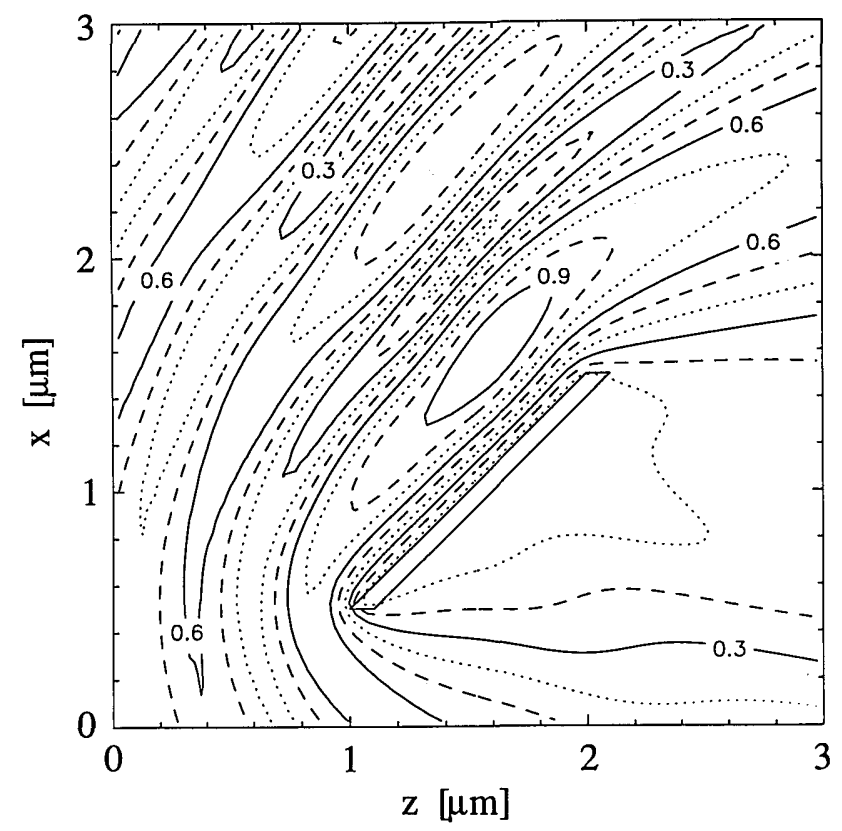

Fig. 7. Interaction of a plane wave in a vacuum $\left(\varepsilon_{\text {ref }}=1\right)$ with a gold plate (thickness $0.1 \mu \mathrm{m}, \varepsilon_{p}=-42.8+i 1.3$ ) oriented at a $45^{\circ}$ angle to the direction of the incoming wave. The spacing between the isoamplitude curves is $10 \%$ of the maximum-minimum amplitude range. The wavelength is $\lambda_{0}=1 \mu \mathrm{m}$, and the mesh size is $\Delta_{x}=\Delta_{z}=0.05 \mu \mathrm{m}$.

cident field is now reflected orthogonally to the surface of the plate. This illustrates the ability of our method to handle arbitrary geometries and nonparaxial propagation situations.

Additional information on these systems can be obtained from the time-averaged Poynting vector $\langle\mathbf{S}\rangle .^{35}$ For the polarization of interest, $\langle\mathbf{S}\rangle$ lies in the $x-z$ plane and can easily be represented. In Fig. 8 we give the timeaveraged Poynting vector corresponding to Figs. 6(a) and 7 , illustrating how the energy flows in these systems. Such information is mandatory for studying the transmission of a signal in integrated structures.

It is important to point out that the boundary conditions are exactly fulfilled by our method. Moreover, as is visible in Figs. 6-9, no energy is reflected at the edges of the computational window. This does not require any special treatment of the boundary points but is automatically taken into account by the formalism underlying the method. Indeed, the nonlocality of Green's function that connects each point in the system to each point of the perturbation guarantees that the boundary conditions are globally fulfilled. This guarantee constitutes a significant advantage of our method.

Since every mesh of the system is connected to all the meshes of the perturbation, the resulting matrix is full. Therefore the CPU time required for obtaining a solution increases significantly with the number of meshes in the perturbation. For the results presented in Fig. 6 the CPU time required on an IBM RISC 6000 model 970 workstation was $14 \mathrm{~s}$ for a $(30 \times 30)$ grid $\left(\Delta_{x}=\Delta_{y}=0.1 \mu \mathrm{m}\right)$, $54 \mathrm{~s}$ for a $(60 \times 60)$ grid $\left(\Delta_{x}=\Delta_{y}=0.05 \mu \mathrm{m}\right)$, and $200 \mathrm{~s}$ for a $(120 \times 120)$ grid $\left(\Delta_{x}=\Delta_{y}=0.025 \mu \mathrm{m}\right)$; these values include the calculation of the Hankel functions required for the computation of $G_{i, j}{ }^{0}$. For small jobs this calculation constitutes the major part of the total required CPU time, and the iterative computation of the field itself took 1,4 , and $19 \mathrm{~s}$, respectively, for the above-mentioned cases.

We now turn our attention to dielectric waveguides. These structures are key components for integrated optics, and our method can handle them accurately. Figure 9 shows the computed field for a monomode symmetrical slab waveguide of $1-\mu \mathrm{m}$ thickness. The core has a dielectric constant of $\varepsilon_{p}=1.1$ and claddings of $\varepsilon_{\text {ref }}=1$. A plane wave of wavelength $\lambda_{0}=1 \mu \mathrm{m}$ was used as the starting field.

The form of the field obtained corresponds to an interference pattern, not to a pure propagating wave, because of the assumption that the perturbation $\varepsilon_{p}$ (in this case
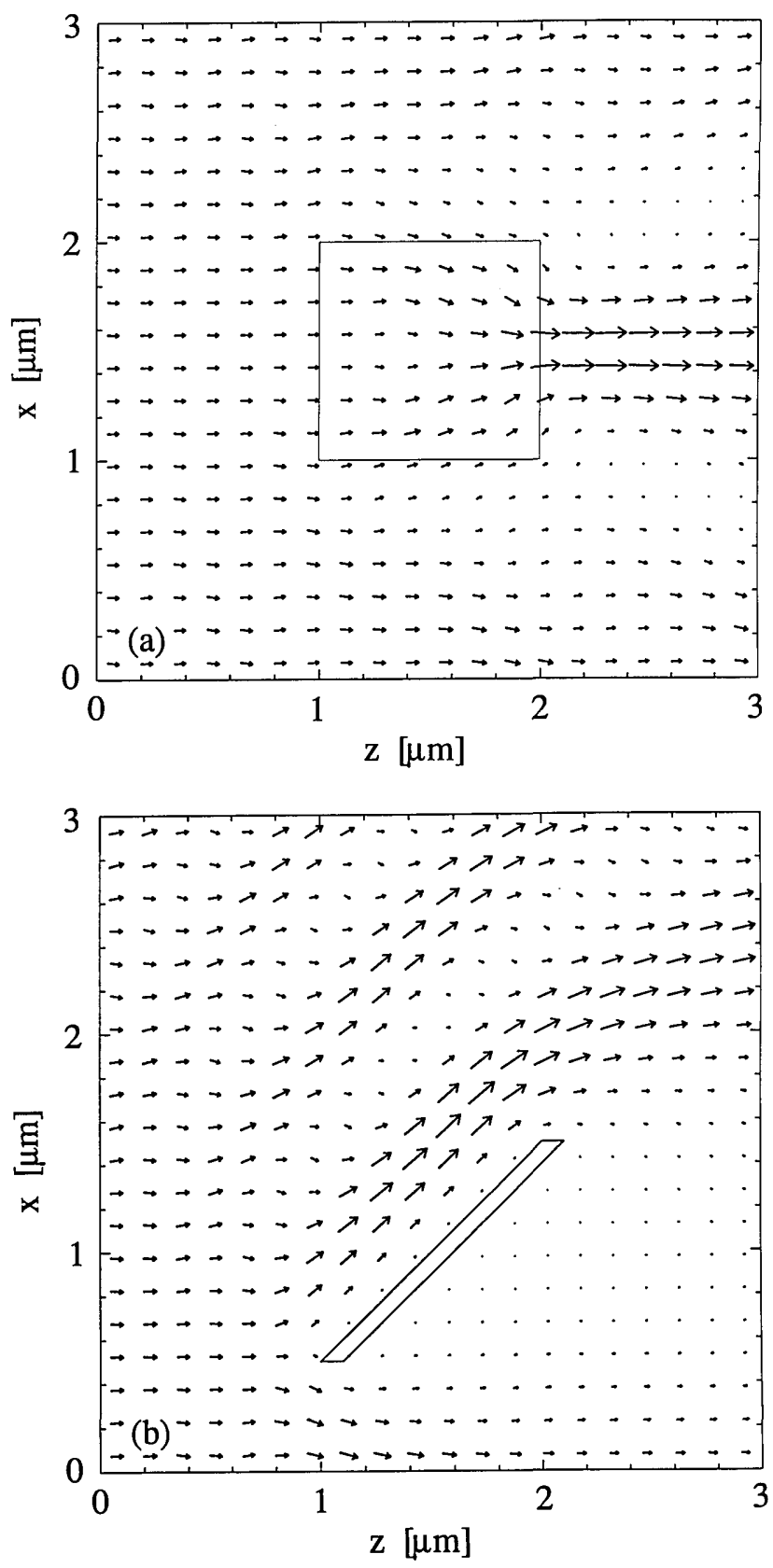

Fig. 8. Representation of the time-averaged Poynting vector $\langle\mathbf{S}\rangle$ corresponding (a) to Fig. 6(a) and (b) to Fig. 7. The orientation and the size of the arrows give the direction and the relative amplitude of $\langle\mathbf{S}\rangle$, respectively. 


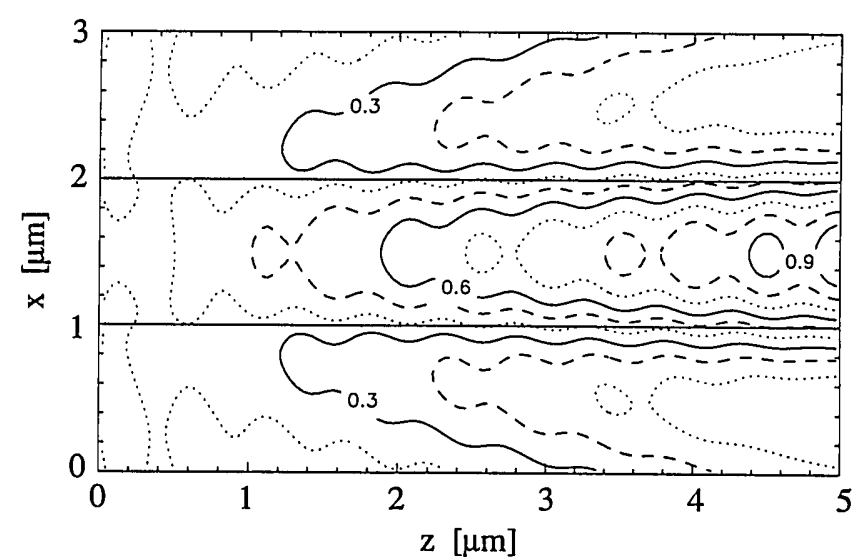

Fig. 9. Field computed for a monomode slab waveguide with a $1-\mu \mathrm{m}$ core of dielectric constant $\varepsilon_{p}=1.1$ and of claddings $\varepsilon_{\mathrm{ref}}=1$. The wavelength is $\lambda_{0}=1 \mu \mathrm{m}$, and the mesh size is $\Delta_{x}=\Delta_{z}=0.05 \mu \mathrm{m}$. The spacing between the isoamplitude curves is $10 \%$ of the maximum-minimum amplitude range.

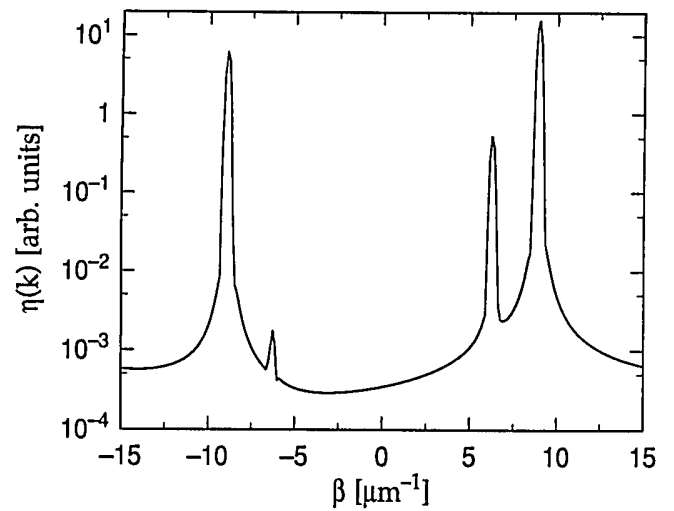

Fig. 10. Mode spectrum of a slab waveguide with a $0.5-\mu \mathrm{m}$ core of dielectric constant $\varepsilon_{p}=2.25$ and of claddings $\varepsilon_{\mathrm{ref}}=1$ for a wavelength of $1 \mu \mathrm{m}$. For this calculation the computational window extended only over the width of the core and was $25 \mu \mathrm{m}$ long in the $z$ direction; the mesh size was $\Delta_{x}=0.025 \mu \mathrm{m}$, and $\Delta_{z}=0.2 \mu \mathrm{m}$

the core of the guide) is embedded into the reference medium $\varepsilon_{\text {ref }}$. Therefore, even if the computational window is limited between $z=0$ and $z=5 \mu \mathrm{m}$, the reference medium extends beyond these limits. This introduces two core-cladding interfaces at $z=0$ and $z=5 \mu \mathrm{m}$ that produce multiple reflections of the field. Such a configuration is close to reality, a real device being generally limited and not infinite. Note that this situation does not correspond to the end of the guide in air but simply to a section of the guide embedded in a homogeneous medium with the same dielectric constant as the claddings.

With this system it is possible to compute the eigenmode spectrum of the waveguide. For this purpose we introduce the integral of the field $\psi(x, z)$ over the width of the guide:

$$
\eta(z)=\int_{-w}^{w} \mathrm{~d} x \psi(x, z)
$$

The $z$ variation of $\eta(z)$ describes the interference pattern of the field (Fig. 9). Since this pattern is due to the interaction of the various modes of the guide, $\eta(z)$ contains information on these modes. Indeed, the Fourier transform $\eta(k)$ of $\eta(z)$ gives the spectrum of the modes that are guided by the structure, as is visible in Fig. 10, where we present $\eta(k)$ for a slab waveguide of core $\varepsilon_{p}=2.25$, cladding $\varepsilon_{\text {ref }}=1$, and thickness $0.5 \mu \mathrm{m}$. For a wavelength of $1 \mu \mathrm{m}$ an infinitely long waveguide with such a dielectric profile has two transverse electric modes of wave numbers $\beta_{0}=8.572 \mu \mathrm{m}^{-1}$ and $\beta_{1}=6.385 \mu \mathrm{m}^{-1}{ }^{30}$ These two modes are clearly visible in Fig. 10 . Because of the interfaces at the ends of the computational window, each eigenmode propagates in the $\pm z$ direction, which doubles the number of peaks in the spectrum, each one appearing with a plus and a minus. The preponderance of the modes with positive $\beta$ is due to the starting field, which had a positive wave number. The opposite result is obtained with a starting field propagating in the $-z$ direction. A completely symmetrical spectrum follows from the use of the superposition of $+z$ and $-z$ propagating starting fields. A fast-Fouriertransform algorithm was used to obtain this spectrum, and a Blackman-Harris window was used to enhance its resolution. ${ }^{39}$ One could also obtain the mode spectrum by simply Fourier transforming $\psi(x, z)$ for a fixed value of $x$. But we have observed that the lateral integration of the field enhances the spectrum and makes the localization of the peaks easier. Because the core of the guide is embedded in a reference medium with the same dielectric constant as the claddings, the reflection of the field at both ends of the computational window occurs only at the interface of the core and the reference medium. No field is reflected at the interface between the cladding and the reference medium. Therefore the ratio of the peak amplitudes corresponding to forwardand backward-propagating modes in Fig. 10 does not give the reflection coefficient for the corresponding mode.

In contrast to the case with the conventional BPM, a very long propagation distance is not required for filtering out the eigenmodes from the starting field. ${ }^{2,3}$ Indeed, we obtained the spectrum presented in Fig. 10 by using a computational window that was $25 \mu \mathrm{m}$ long and as large as the core of the guide; the mesh sizes were $\Delta_{x}=$ $0.025 \mu \mathrm{m}$ and $\Delta_{z}=0.2 \mu \mathrm{m}$. A wider computational window (extending to the claddings of the guide) yielded the same results. Hence computing only the field in the core (i.e., in the perturbation) gives sufficient information on the various guided modes of the structure. For the results presented in Fig. 10, we needed $10 \mathrm{~min}$ to compute $G_{i, j}^{0}$ and $47 \mathrm{~min}$ to compute the field iteratively on an IBM RISC 6000 model 970 workstation.

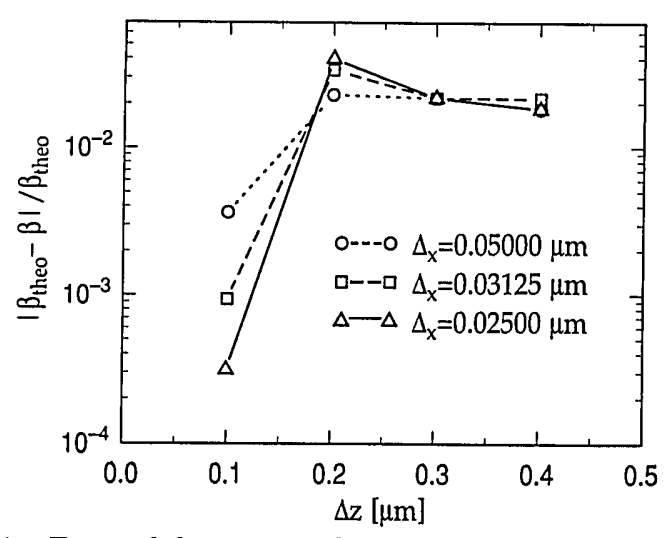

Fig. 11. Error of the computed wave number $\beta$ relative to its theoretical value $\beta_{\text {theo }}$ as a function of the mesh size. 
One can obtain the exact value of the wave numbers $\beta$ accurately by fitting a cubic polynomial to the corresponding peak of the spectrum. ${ }^{40}$ This value depends on the mesh size used for the computation. In Fig. 11 we present the error of the computed wave number $\beta_{0}$ relative to the theoretical value $\beta_{\text {theo }}=8.572 \mu \mathrm{m}^{-1}$ for different mesh sizes $\Delta_{x}$ and $\Delta_{z}$ and for the abovementioned computational window. These results show that the dominant parameter affecting the accuracy of the wave number is the mesh size along the $z$ direction. For small values of $\Delta_{z}$ the value of $\Delta_{x}$ also influences the exactness of the results (Fig. 11). It is important to emphasize that accurate results are obtained despite the very large step of the dielectric constant between the core and the cladding of the guide. Again the relation between the accuracy of the results and the mesh size depends on the effective wavelength in the core. For example, a guide with the same cladding $\left(\varepsilon_{\text {ref }}=1\right)$ but a smaller step of dielectric constant $\left(\varepsilon_{p}=1.05\right)$ yields values one order of magnitude more accurate with the same numerical parameters than in Fig. 11.

\section{CONCLUSIONS}

We have presented a new approach to the computation of the electrical field propagating in dielectric media. Using the Green's function technique, we have found that our method yields the exact solution of the wave equation. No approximations regarding the form of the field are required, and we showed that situations far beyond the scope of the conventionally used paraxial approximation can be simulated with this method. Dielectric media as different as air, semiconductors, and metal can be simulated, and no restrictions are imposed on the variations of the dielectric function in the system under study.

The boundary conditions in the system and at the edges of the computational window are exactly fulfilled by our method, and no radiated energy is reflected back into the system.

An efficient and extremely stable iterative numerical scheme was proposed. The influence of the numerical parameters on the accuracy of the results was studied, and we showed that extremely accurate results can be obtained with use of an appropriate discretization refinement.

Application of the method to the determination of the spectrum of eigenmodes of a waveguide was discussed in detail.

Finally, we believe that our iterative scheme based on the parallel use of Lippman-Schwinger's and Dyson equations is relevant to other problems in numerical physics. The study of its application to the computation of the field susceptibility of a composite atomic system is in progress. ${ }^{41}$

\section{REFERENCES}

1. M. D. Feit and J. A. Fleck, Jr., "Light propagation in gradedindex optical fibers," Appl. Opt. 17, 3990-3998 (1978).

2. M. D. Feit and J. A. Fleck, Jr., "Calculation of dispersion in graded-index multimode fibers by a propagating-beam method," Appl. Opt. 18, 2843-2851 (1979).

3. M. D. Feit and J. A. Fleck, Jr., "Computation of mode properties in optical fiber waveguides by a propagating beam method," Appl. Opt. 19, 1154-1164 (1980).
4. J. van Roey, J. van der Donk, and P. E. Lagasse, "Beampropagation method: analysis and assessment," J. Opt. Soc. Am. 71, 803-810 (1981).

5. L. Thylén, "The beam propagation method: an analysis of its applicability," Opt. Quantum Electron. 15, 433-439 (1982).

6. D. Yevick and B. Hermansson, "Split-step finite difference analysis of rib waveguides," Electron. Lett. 25, 461462 (1989).

7. Y. Chung and N. Dagli, "An assessment of finite difference beam propagation method," IEEE J. Quantum Electron. 26, 1335-1339 (1990).

8. T. B. Koch, J. B. Davies, and D. Wickramasinghe, "Finite element/finite difference propagation algorithm for integrated optics," Electron. Lett. 25, 514-516 (1989).

9. M. Matsuhara, "A novel beam propagation method based on the Galerkin method," Electron. Commun. Jpn. 73, 4147 (1990).

10. J. Gerdes and R. Pregla, "Beam-propagation algorithm based on the method of lines," J. Opt. Soc. Am. B 8, 389394 (1991).

11. S. T. Chu and S. K. Chaudhuri, "A finite-difference time-domain method for the design and analysis of guidedwave optical structures," IEEE J. Lightwave Technol. 7, 2033-2038 (1989).

12. D. Yevick and M. Glasner, "Forward wide-angle light propagation in semiconductor rib waveguides," Opt. Lett. 15, 174-176 (1990).

13. A. Splett, M. Majd, and K. Petermann, "A novel beam propagation method for large refractive index steps and large propagation distances," IEEE Photon. Technol. Lett. 3, 466-468 (1991).

14. G. R. Hadley, "Wide-angle beam propagation using Padé approximant operators," Opt. Lett. 17, 1426-1428 (1992).

15. L. Thylén and C. M. Lee, "Beam-propagation method based on matrix diagonalization," J. Opt. Soc. Am. A 9, 142146 (1992)

16. M. S. Stern, "Rayleigh quotient solution of semivectorial field problems for optical waveguides with arbitrary index profiles," IEEE Proc. J 138, 185-190 (1991).

17. W. P. Huang, S. T. Chu, and S. K. Chaudhuri, "A semivectorial finite-difference time-domain method," IEEE Photon. Technol. Lett. 3, 803-806 (1991).

18. P. L. Liu and B. J. Li, "Semivectorial beam-propagation method for analyzing polarized modes of rib waveguides," IEEE J. Quantum Electron. 28, 778-782 (1992).

19. R. Clauberg and P. von Allmen, "Vectorial beam-propagation method for integrated optics," Electron. Lett. 27, 654655 (1991).

20. W. P. Huang, C. L. Xu, S. T. Chu, and S. K. Chaudhuri, "A vector beam propagation method for guided-wave optics," IEEE Photon. Technol. Lett. 3, 910-913 (1991).

21. O. J. F. Martin, R. Clauberg, and P. von Allmen, "Demonstration of the three-dimensional vectorial beam propagation," presented at the European Conference on Optical Communication, Paris, September 9-12, 1991.

22. W. Huang, C. Xu, S. T. Chu, and S. K. Chaudhuri, "The finite-difference vector beam propagation method: analysis and assessment," IEEE J. Lightwave Technol. 10, 295305 (1992).

23. Y. Chung, N. Dagli, and L. Thylén, "Explicit finite difference vectorial beam propagation method," Electron. Lett. 27, 2119-2120 (1991).

24. J. M. Liu and L. Gomelsky, "Vectorial beam propagation method," J. Opt. Soc. Am. A 9, 1574-1585 (1992).

25. E. W. Kolk, N. H. G. Baken, and H. Blok, "Domain integral equation analysis of integrated optical channel and ridge waveguides in stratified media," IEEE Trans. Microwave Theory Tech. 38, 78-85 (1990).

26. N. H. G. Baken, M. B. J. Diemeer, J. M. Van Splunter, and $\mathrm{H}$. Blok, "Computational modeling of diffused channel waveguides using a domain integral equation," IEEE J. Lightwave Technol. 8, 576-586 (1990).

27. H. J. M. Bastiaansen, N. H. G. Baken, and H. Blok, "Domainintegral analysis of channel waveguides in anisotropic multilayered media," IEEE Trans. Microwave Theory Tech. 40, 1918-1926 (1992). 
28. C. Girard and X. Bouju, "Self-consistent study of dynamical and polarization effects in near-field optical microscopy," J. Opt. Soc. Am. B 9, 298-305 (1992).

29. A. Dereux and D. W. Pohl, "The $90^{\circ}$ prism as a model SNOM probe: near-field, tunneling, and light scattering properties," in Proceedings of the NATO Advanced Research Workshop on Near Field Optics, D. W. Pohl, ed. (Kluwer, Dordrecht, The Netherlands, 1993), pp. 189-198.

30. D. Marcuse, Theory of Dielectric Optical Waveguides (Academic, San Diego, Calif., 1974).

31. E. N. Economou Green's Functions in Quantum Physics, 2nd ed. (Springer-Verlag, Berlin, 1990).

32. G. Arfken, Mathematical Methods for Physicists, 3rd ed. (Academic, San Diego, Calif., 1985).

33. A. D. Yaghjian, "Electric dyadic Green's functions in the source region," Proc. IEEE 68, 248-263 (1980).

34. G. G. O'Brien, M. A. Hyman, and S. Kaplan, "A study of the numerical solution of partial differential equations," J. Math. Phys. 29, 223-251 (1951)

35. M. Born and E. Wolf, Principles of Optics, 6th ed. (Pergamon, Oxford, U.K., 1980).
36. C. Cohen-Tannoudji, B. Diu, and F. Laloe, Quantum Mechanics (Hermann, Paris, 1977).

37. D. W. Pohl, "Nano-optics and scanning near-field optical microscopy," in Scanning Tunneling Microscopy II, R. Wiesendanger and H. J. Güntherodt, eds. (Springer-Verlag, Berlin, 1992), pp. 233-271.

38. J. D. Jackson, Classical Electrodynamics, 2nd ed. (Wiley, New York, 1975).

39. F. J. Harris, "On the use of windows for harmonic analysis with the discrete Fourier transform," Proc. IEEE 66, 5183 (1978).

40. G. N. Kamm, "Computer Fourier-transform techniques for precise spectrum measurements of oscillatory data with applications to the de Haas-van Alphen effect," J. Appl. Phys. 49, 5951-5970 (1978).

41. C. Girard, A. Dereux, and O. J. F. Martin, "Field susceptibility of a composite system: application to van der Waals dispersive interactions inside a finite line of physisorbed atoms," Surf. Sci. 295, 445-456 (1993). 